\title{
Tratamiento informativo de la pandemia del coronavirus en los medios digitales españoles
}

\author{
Natalia Abuín Vences \\ Javier Sierra Sánchez \\ LUIS MaÑas-VINIEgRA \\ Patricia NúÑez Gómez \\ Universidad Complutense de Madrid Universidad Complutense de Madrid Universidad Complutense de Madrid Universidad Complutense de Madrid \\ nabuinve@ccinf.ucm.es \\ javier_sierra@ucm.es \\ Imanas@ucm.es \\ pnunezgo@ccinf.ucm.es \\ https://orcid.org/0000-0002-4153-9390 https://orcid.org/0000-0001-8572-7564 https://orcid.org/0000-0001-9129-5673 https://orcid.org/0000-0002-0073-2020
}

\section{Coronavirus pandemic news coverage in spanish digital media}

\section{RESUMEN}

El presente trabajo de investigación empírica aborda la cobertura informativa del SARS-CoV-2 en los medios digitales españoles. Se analizan las piezas informativas que mayor alcance/engagement han obtenido en medios digitales con

la finalidad de conocer cuáles han sido las variables más destacadas en el discurso de la pandemia. Del mismo modo, se ha realizado un análisis del discurso analizando el tono empleado. Los resultados apuntan que las temáticas más destacadas en los titulares de lo medios digitales han sido la sanidad, la política y la economía. Son estas tres temáticas las que más titulares con tono positivo y negativo han recibido. La variable tono ha sido, a nivel global, balanceada por igual en la muestra total de análisis (mismo número de titulares positivos y negativos). La sociedad civil ha sido el actor informativo que ha acaparado mayor interés en los titulares

como objeto protagonista de la información. Al cruzar la variable tono con los actores informativos se ha podido comprobar que la sociedad civil y el Gobierno son los actores que han atraído el mayor número de titulares con tono negativo y positivo. Se ha profundizado en las temáticas que han provocado esa dualidad.

PALABRAS CLAVE

Medios digitales; Coronavirus; COVID-19; Engagement; Noticias.

\section{ABSTRACT}

This empirical research paper deals with SARS-CoV-2 news coverage (initial outbreak and expansion) in Spanish digital media. News items published in digital media that have reached furthest on scope/engagement are analyzed with the aim of learning which have been the most outstanding variables in the pandemic discourse. Similarly, a discourse analysis was carried out by looking into the tone employed in relation to the aforementioned variables. Findings reveal that health, politics and economics have been the most prominent topics in digital media headlines. These three themes have received the most headlines with both positive and negative tones. Globally, the tone variable has been equally balanced in the total analysis samples (same number of positive and negative headlines). Civil society has been the most newsworthy subject in capturing headlines as the main news' protagonist. On crossing the tone variable with the news' actants, we have been able to verify that civil society and Government are the subjects that have attracted the most headlines either with negative or positive tones. Further work has been done on the themes that have caused this duality.

KEYWORDS

Digital media; Coronavirus; COVID-19; Engagement; News. 


\section{Introducción}

Los entornos digitales han democratizado el acceso a la información de la población mundial. Esto ha sido provocado por varios factores, entre los que cabe destacar: la aparición de medios sociales, la diversidad de canales (Hernández-Pérez y Rodríguez Mateos, 2016), la multiplicidad de dispositivos y la aparición de otros nuevos como tabletas, teléfonos y smartphones (Jenkins, 2006; Campos-Freire, 2010), la socialización del consumo informativo (Martínez Costa, Sánchez Blanco y Serrano Puche, 2018) y la velocidad de circulación de los contenidos en tiempo real (Guallar, 2011). Culver y Jacobson (2012, p.8) señalan que las tecnologías han provocado una nueva comunicación más sencilla, accesible e instantánea. Casero-Ripollés (2020) apunta en una investigación reciente sobre la pandemia de la COVID-19 que, en situaciones críticas de alta complejidad y riesgo para la vida humana, como el brote del nuevo coronavirus, los ciudadanos sitúan la búsqueda de información y el seguimiento de las noticias como actividades clave. Así, en los inicios del brote, el consumo de noticias se incrementó un 62\%.

El portal Economía Digital (2020) indica que en los buscadores la tendencia es clara, con un incremento del 252\% en las búsquedas vinculadas a la palabra "coronavirus", siendo la Comunidad de Madrid la que mayor cantidad concentra debido al elevado número de infectados, con más de 70.000 positivos en mayo de 2020.

Casero-Ripollés (2020, p.2) señala que, desde la declaración de emergencia nacional ante la crisis sanitaria, la información sobre el brote se ha convertido en un bien preciado y valioso para hacer frente a la situación. De este hecho ha dejado constancia ABC (2020), cuando recoge los datos de un estudio de Comscore en el que se indica que el confinamiento ha disparado el uso de internet, siendo el consumo de noticias digitales la categoría que más sube: entre la semana del 13 al 19 de enero y la del 23 al 29 de marzo de 2020, creció un 59\% en España. Otro estudio de la consultora Ymedia del que se hace eco el portal Marketing News (2020) señala que se produjo un incremento del 30\% en el número de usuarios que accedieron a los contenidos web de los diarios durante el confinamiento.

Estos datos podrían hacer pensar que los medios tradicionales están atravesando una difícil etapa. De hecho, varios estudios confirman la bajada de consumo de noticias de medios offline (Labella, 2012; Amoedo, Vara-Miguel y Negredo, 2018). Esta crisis sanitaria ha provocado un importante crecimiento del consumo de televisión y de medios digitales, tal y como se hace eco Europa Press (2020) a partir de un informe de IPG
Mediabrands, de tal manera que ambos medios offline y online han aumentado considerablemente desde que se decretó el estado de alarma y el confinamiento obligatorio. Las versiones digitales de los medios de comunicación informativos son las que más están notando este cambio de hábitos en el consumo: reciben un $45 \%$ más de páginas vistas y han incrementado su tráfico un 100\%, la audiencia digital de la radio online crece en un $112 \%$ y la televisión online en directo suma un 93\% de usuarios únicos. Por su parte, la televisión se ha convertido también en la fuente principal de entretenimiento e información. A los medios de comunicación tradicionales no les ha quedado más remedio, tal y como señalan Hernández Pérez y Rodríguez Mateos (2016), que adaptarse para que sus "productos" y sus noticias puedan ser consumidas a través de dispositivos móviles y en plataformas tecnológicas que no son propias y en las que se distribuye su contenido, como Facebook, Instagram, Twitter, YouTube, WhatsApp, Snapchat y tantas otras. De hecho, incluso los medios escritos están produciendo o comprando gráficos, vídeos e interactivos que hagan el contenido más atractivo para poder hacerse un hueco en medios sociales.

\subsection{El consumo de noticias en medios digitales}

Este trabajo se centra en el análisis de los titulares de las noticias en medios digitales online que han tenido mayor alcance durante la crisis sanitaria en España, de ahí que sea necesario aportar algunas cifras de interés de lo que supone el consumo de información en medios cuyo soporte es digital. Según el Informe Digital News Report 2018 (Amoedo, Vara-Miguel y Negredo, 2018) el $64 \%$ de los usuarios en España se informan con algún medio nativo digital. El 48\% de los adultos usa Facebook para leer, ver, encontrar, compartir o comentar noticias a lo largo de la semana; el 36\% emplea WhatsApp para ello y lo hace de forma homogénea por edades. Pero los usuarios no solo consumen de forma pasiva la información (consumidor) sino que pasan a ser prosumidores y prodiseñadores (Serrano et al., 2017), tal y como indica el informe Digital News Report 2018, elaborado en colaboración con el Reuters Institute for the Study of Journalism. Los usuarios españoles de noticias online no se limitan a consumir información en internet, sino que interactúan de alguna manera con ella. Algo más de la mitad (el 52\%) de los usuarios en España comparten noticias al menos una vez a la semana, el 32\% de los internautas adultos que consumen noticias online dicen comentarlas, ya sea en sus perfiles en redes sociales (26\%) o, en menor medida, en los sitios web de los medios de comunicación (11\%). Puntuar noticias o mar- 
carlas con "me gusta" o como "favorito" es una actividad habitual para el $29 \%$ de los encuestados.

Estos datos hacen ver una necesaria convivencia y complementariedad (Dutta-Bergman, 2004) entre la prensa tradicional y la digital en la que las redes sociales se convierten en facilitadoras, potenciando el acceso a este tipo de contenidos. Hay varios estudios que han comprobado una correlación positiva entre el uso de redes y el acceso de noticias que han denominado como acceso incidental de las mismas (Lee, 2009; Effing, Van Hillegersberg y Huibers, 2011; Oeldorf-Hirsch y Sundar, 2015; Boczkowski, Mitchelstein y Matassi, 2018; Newman et al., 2019). Los medios de comunicación utilizan Twitter y Facebook con el fin de promover sus contenidos y derivar tráfico a su web. Si bien es cierto que el acceso a redes no reemplaza a los medios, ha causado una transformación en los procesos de producción y consumo de los contenidos informativos (Fernández-Medina, Proust y Núñez-Mussa, 2018).

Este afán de los medios por atraer tráfico hacia sus espacios web, un factor fundamental para poder financiarse a través de la venta de espacios publicitarios, provoca el fenómeno del clickbait, también conocido como ciber-cebo. Algunos medios basan su estrategia para generar tráfico web en la creación de titulares sensacionalistas, cuya finalidad es atraer clics y fomentar el envío de dicho material a través de las redes sociales. Aprovechan la curiosidad del lector para que pinche en el titular. Para ello, le proporcionan los suficientes datos como para despertar su apetito, pero la única manera de satisfacerlo es acceder al contenido. Hay que tener en cuenta que el titular es el foco del artículo, porque es lo primero que ve la audiencia. Para atraer la atención se enfatiza la parte más interesante del texto para invitar al usuario a que siga leyendo el contenido. La función principal del titular es dar al lector un mensaje importante sobre el artículo, por lo que debe condensar la idea principal del contenido. Sin embargo, esto no siempre es así. Algunos encabezados no proporcionan un resumen de la noticia, sino que se centran en la parte que debe ser destacada o promocionada, con la finalidad de atraer la atención del lector, aunque esta sea una parte irrelevante del mismo (Liang y Wu, s.f.).

En este sentido, tampoco se debe olvidar la relación entre la emoción y la viralidad: los mensajes que despiertan emociones, ya sean positivas o negativas, suelen obtener un mayor alcance que los neutrales (Berger y Milkman, 2012).

Todos estos datos permiten afirmar que los medios digitales y la interacción de los usuarios con los contenidos informativos a través de estos han proporcionado un nuevo sentido a la Teoría de los Usos y Gratificaciones, para explicar por qué los usuarios prefieren determinados contenidos frente a otros en entornos digitales, además de poner en valor lo que hoy es una realidad incuestionable: las audiencias activas, de las que ya se hablaba en los albores de esta teoría.

\subsection{La Teoría de los Usos y Gratificaciones aplicada a los medios digitales}

El origen de la Teoría de los Usos y Gratificaciones (en adelante UyG) se remonta a los años treinta del siglo $X X$, en los inicios de la investigación empírica relacionada con la comunicación de masas. Uno de los principales objetos de estudio en aquel momento eran los efectos que los medios de masas tenían sobre las audiencias, pero pronto se comenzaría a investigar qué motivos empujan a los individuos a consumir determinados medios.

En 1973, Katz, Blumler y Gurevitz formulan la UyG, que postula la audiencia como un desafío para los productores de información, indicando que las gratificaciones de las audiencias se derivan, al menos, de tres fuentes diferentes: contenido del medio, exposición al mismo y contexto social. La UyG explica los orígenes sociales y psicológicos de las necesidades que impulsan la exposición y la selección de los medios para satisfacer las necesidades individuales de la audiencia (Katz, Blumler y Gurevitch, 1973). Este enfoque sostiene que los individuos son usuarios activos de medios y orientados a objetivos, sus motivaciones explican la exposición a los medios, pues los eligen para satisfacerlas (Blumler, 1979; Katz, Haas y Gurevitch, 1973). Arraigado en este enfoque, el concepto de la necesidad de orientación ofrece una explicación psicológica de por qué las personas buscan información en los medios: necesitan familiarizarse con el entorno a través de los medios de comunicación (Matthes, 2005; Weaver, 1980).

Esta arraigada teoría cobra especial importancia con la llegada de los medios digitales, que modifican completamente la relación con la audiencia, que ahora puede comentar y compartir sus contenidos, pudiendo viralizarse sus opiniones en pocas horas. Esto ha provocado que el concepto de audiencia activa alcance una nueva dimensión. Esta noción fue propuesta por los investigadores pioneros de los usos y gratificaciones para capturar el propósito y la atención en el consumo de medios y contrastarlo con el supuesto general de una "audiencia pasiva" entre los efectos mediáticos (Rubin, 2009). Con la llegada de los medios digitales se ha convertido en una realidad obvia (Sundar y Limperos, 2013). Según estos mismos autores, las audiencias de Internet son tan activas que nos referimos a ellas como usuarios, 
en sintonía con el espíritu del paradigma de los usos y gratificaciones.

Respecto a las audiencias activas, en los medios digitales deben distinguirse dos tipos: la social y la creativa. Podemos definir la audiencia social como aquella que utiliza simultáneamente dos pantallas o dispositivos (Deltell, 2014). El concepto de audiencia creativa fue acuñado por el sociólogo Manuel Castells en 2011 para definir aquella parte de la audiencia social que interactúa y crea opinión. Un nuevo modelo de audiencia que surge con Internet y muy especialmente con las redes sociales. El espectador deja de ser un mero lector pasivo de textos audiovisuales para convertirse en un creador de respuestas y textos transmedia.

\subsection{Audiencia, alcance y engagement}

Una de las grandes ventajas de los medios digitales frente a los mass media tradicionales es que permiten que el consumo de productos informativos o de entretenimiento se pueda producir en cualquier momento, gracias a la banda ancha móvil, o desde cualquier lugar, a través de los dispositivos conectados, por lo que parece oportuno pasar a medir el consumo en términos de alcance y engagement, en lugar de hablar de audiencia. El alcance hace referencia al número de personas distintas que ven un contenido, es el promedio de la audiencia efectiva. En otras palabras, este dato se refiere a usuarios únicos que han visto una publicación.

Para entender el concepto de alcance aplicado al consumo de contenidos informativos en medios digitales, debemos retomar la UyG, y la idea de las audiencias activas, según la cual, los usuarios eligen los medios de manera deliberada y discriminatoria (Mersey, Malthouse y Calder, 2010). Ya en 1987, Stone indicó que las personas no atienden mensajes que no tienen un interés para ellos. Elegirán aquel contenido que ofrezca los elementos que consideren valiosos, incluso si ese valor solo consiste en un placer momentáneo.

Cuando la noticia es capaz de generar una audiencia creativa, hablamos de engagement y participación. La investigación sobre los procesos de engagement indican que es inherentemente social, relacional e interactivo por naturaleza (Dessart, 2017). La definición del término también conlleva aspectos cognitivos, emocionales y de comportamiento (Gambetti, Graffigna y Biraghi, 2012). Bowden (2009) además de la interactividad añade la co-creación y la multidimensionalidad, es decir, da lugar a una audiencia que comenta, interactúa y genera opinión.

Mersey, Malthouse y Calder (2010) definen el engagement como el resultado de un cúmulo de experiencias del lector con el medio digital en cuestión. Demostraron, además, que hay dos tipos de engagement: el personal y el social-interactivo. El primero se refiere a percepciones personales del tipo "este sitio me hace pensar en cosas nuevas" o "este sitio a menudo me proporciona algo de qué hablar" que puede aparecer también en los medios de masas tradicionales. El segundo es propio de los medios digitales y proporciona elementos experienciales del tipo "este sitio me permite socializar" y "contribuyo a la conversación en este sitio".

En este sentido, hay que considerar también cómo circulan las noticias en el entorno digital, pues influye en términos de alcance, ya que las audiencias online pueden acceder a los contenidos más fácilmente, tanto a las noticias globales como a las locales.

De un esquema comunicacional de masas, que va desde un emisor (o un grupo pequeño de emisores) a múltiples receptores dispersos y mayormente incomunicados entre sí, pasamos a una estructura en red en la que cada ciudadano representa un nodo que es capaz de recibir, compartir, retocar y construir nueva información. Según Mitchelstein y Boczkowski (2018) accedemos a esta información de manera breve, interrumpida y parcial: su consumo es efímero. La cuestión es comprender qué tipo de contenidos atraen la atención de las audiencias digitales, para entender cuáles son las que consiguen mayor alcance/engagement en momentos de crisis, como es el caso de la COVID-19.

\section{Objetivos}

A partir de una investigación longitudinal del análisis de los titulares de los medios digitales que más alcance/ engagement han tenido, el objetivo general de este trabajo es determinar qué tipo de noticias han obtenido mayor alcance/engagement durante la crisis de la COVID-19 con la finalidad de conocer los temas, actores informativos y el tipo de titulares sobre la crisis sanitaria que han protagonizado las noticias digitales. De este objetivo general, se desprenden los siguientes objetivos específicos:

- Examinar los titulares de las noticias digitales que más alcance/engagement han generado con el objeto de conocer en profundidad qué temas y qué finalidad (tono) presentan.

- Estudiar el actor y el espacio informativo del titular de las noticias objeto de estudio para descubrir qué tipología tiene más presencia en esta pandemia.

- Llevar a cabo un análisis discursivo de los titulares para establecer posicionamientos. 
- Definir la realidad proyectada por las noticias de mayor alcance para establecer la taxonomía temática de cada uno de los medios objeto de estudio.

\section{Metodología}

Para alcanzar estos objetivos, se ha planteado una investigación longitudinal (desde el 8 de marzo al 27 de abril 2020) de las noticias digitales relacionadas con la COVID-19 que obtuvieron mayor alcance/engagement durante el periodo objeto de estudio. Para seleccionar las noticias, se utilizó la herramienta Buzzsumo, que permite localizar los contenidos digitales con mayor alcance/engagement en función de los parámetros de búsqueda introducidos por los usuarios. En primer lugar se introdujo un parámetro de selección semántico: los titulares de las noticias objeto de estudio debían contener la palabra COVID-19 o coronavirus. Así mismo, se aplicaron filtros geográficos (España), temporales (marzo-abril) con el fin de extraer la información objeto de estudio. La muestra final está compuesta por un total de 200 noticias vinculadas mediante sus titulares a la COVID-19 o al coronavirus (100 noticias cuyos titulares contenían la palabra COVID-19 y otras 100 con la palabra coronavirus), publicadas en los meses de marzo y abril, lo que permite analizar el tipo de medio y el reclamo utilizado por los mismos para atraer la atención de los usuarios. Es necesario resaltar que la muestra se seleccionó en base al alcance (contenidos digitales más vistos por los usuarios) y el engagement (noticias que han generado más comentarios y han sido compartidas por los usuarios). Al seleccionar las noticias objeto de estudio en función del alcance/engagement y no de las audiencias tradicionales, en la muestra no aparece ninguno de los grandes medios digitales como El País o El Mundo, pero sí medios regionales como La Voz de Galicia o Faro de Vigo, lo que se relaciona directamente con la Teoría de los Usos y Gratificaciones: uno de los criterios de elección de los contenidos por parte de los usuarios de medios digitales es la necesidad familiarizarse con el entorno (Matthes, 2005; Weaver, 1980).

En cuanto a la metodología, se llevó a cabo una análisis de contenido descriptivo cuantitativo y cualitativo, apoyado en las herramientas de big data que ofrece la aplicación Buzzsumo utilizada para seleccionar la muestra. Se obtuvieron datos pormenorizados (diarios, semanales y mensuales) para conocer el alcance de esta pandemia mundial sin precedentes. También se buscaba conocer el tono o dirección de los titulares de las noticias que mayor alcance y engagement han generado en la población. En este sentido, se estableció la codificación de titular con predominio de frases positivas, negativas o ambivalentes sobre la pandemia (Bardin, 1986; López-Aranguren, 2016; Piñuel y Gaitán, 1995).

Los hallazgos potenciales de las noticias que hacían referencia a la crisis sanitaria se procesaron siguiendo procedimientos de investigación del análisis de contenido (Berelson, 1952; Martín López, 1963; Krippendorff, 1990). A partir de esta metodología, se estableció un sistema categorial consistente en clasificar la información que contenían las noticias partir de un criterio previamente definido. Las noticias fueron codificadas en función de las siguientes variables:

- Sitio de origen de cada noticia: diarios/portales de información con dominio/sede en España.

- Redes sociales por las que circularon las noticias para conocer los medios con más alcance/engagement: Facebook, Twitter, Pinterest y Reddit.

- Tema: crisis sanitaria a partir de los dos términos objeto de análisis (COVID-19 y Coronavirus).

- Muestra: las noticias con más alcance/engagement por término analizado. Noticias referidas al término Coronavirus (100) y al término COVID-19 (100).

- Tema principal: Política, Economía, Empleo, Internacional, Sanidad, Educación, Unión Europea, Medio ambiente, Energía, Deportes, Industria, Bienestar social.

- Actor informativo: institucionales/oficiales, políticos, económicos, alternativos, sociedad civil, sistema de medios de comunicación, culturales, otros.

- Espacio informativo: España, referencia global/mundial, Estados Unidos, Unión Europea, Resto de Europa, Rusia, Asia, América Latina, Oriente medio y Magreb, África Subsahariana.

- Tono del titular: positivo, negativo y neutro.

Fundamentalmente, se ha empleado un método cuantitativo, descriptivo y longitudinal, empleando tan solo la variable cualitativa para el análisis del tono del titular de las noticias que más alcance habían tenido en el cómputo global. El análisis cualitativo se realizó con el software Atlas.ti v. 8.4.4, mientras que para el análisis estadístico se utilizó SPSS v.25.

\section{Resultados}

El enfoque del tema de los titulares (Tabla 1) se posiciona fundamentalmente en los aspectos sanitarios de la crisis (61,5\%), seguido de temas políticos (16,5\%) y la preocupación a nivel económico de la pandemia 
(11\%). Es necesario recordar que los titulares corresponden al primer mes y medio de la crisis, donde el interés informativo de los usuarios se centraba en los datos de evolución de la pandemia, por lo que es normal que sean este tipo de titulares los que hayan conseguido un mayor alcance/engagement, seguidos a gran distancia por los temas políticos y económicos, que han ido recobrando protagonismo a medida que iba remitiendo la crisis sanitaria. Estos datos reflejan la necesidad de orientación postulada por la Teoría de Usos y Gratificaciones: durante el confinamiento, una de las únicas formas de relación con el entorno fue el consumo de noticias. Los usuarios completamente confinados buscaban en la información y las noticias digitales el contacto con el exterior.

\begin{tabular}{|c|c|c|}
\cline { 2 - 3 } \multicolumn{1}{c|}{} & Frecuencia & Porcentaje \\
\hline $\begin{array}{c}\text { Bienestar social } \\
\text { (políticas sociales) }\end{array}$ & 4 & $2,0 \%$ \\
\hline Deportes & 1 & $0,5 \%$ \\
\hline Economía & 22 & $11,0 \%$ \\
\hline Empleo & 6 & $3,0 \%$ \\
\hline Industria & 3 & $1,5 \%$ \\
\hline Internacional & 1 & $0,5 \%$ \\
\hline Medio Ambiente & 2 & $1,0 \%$ \\
\hline Monarquía & 1 & $0,5 \%$ \\
\hline Poder Judicial & 3 & $1,5 \%$ \\
\hline Política & 33 & $16,5 \%$ \\
\hline Religión & 1 & $0,5 \%$ \\
\hline Sanidad & 123 & $61,5 \%$ \\
\hline Total & $\mathbf{2 0 0}$ & $100,0 \%$ \\
\hline
\end{tabular}

Tabla 1. Tema de los titulares. Elaboración propia.

También se deseaba conocer el tono o dirección de los titulares. Se codificó el titular destacado de portada con predominio de frases positivas, negativas o neutras sobre la pandemia de la COVID-19. En la tabla 2, referida al tono de los titulares, puede apreciarse una igualdad entre el tono positivo y negativo empleado en el uso de los titulares.

\begin{tabular}{|c|c|c|}
\cline { 2 - 3 } \multicolumn{1}{c|}{} & Frecuencia & Porcentaje \\
\hline Negativo & 71 & $35,5 \%$ \\
\hline Neutro & 58 & $29,0 \%$ \\
\hline Positivo & 71 & $35,5 \%$ \\
\hline Total & $\mathbf{2 0 0}$ & $\mathbf{1 0 0 , 0} \%$ \\
\hline
\end{tabular}

Tabla 2. Finalidad/tono empleado en los titulares. Elaboración propia.
Como se puede observar, un 71\% de los titulares con mayor alcance/engagement son positivos o negativos. La explicación a este fenómeno radica en la relación existente entre la emoción y la viralidad (Berger y Milkman, 2011): los mensajes que despiertan emociones, ya sean positivas o negativas, suelen obtener un mayor alcance que los neutrales.

Ambos tipos de titulares se caracterizan por la utilización de adjetivos calificativos (inflado, raro, ejemplar...) y adverbios de afirmación o negación. El tipo de verbo empleado también ha ayudado a clasificar de manera objetiva el tono de los titulares (denunciar, permitir, obligar...). La mayoría de los titulares positivos objeto de estudio están relacionados el descenso en el número de decesos a causa de la pandemia o el relajamiento de las medidas de confinamiento por parte del Gobierno. Respecto a los negativos, hay que resaltar que están relacionados con el fallecimiento de individuos o con los fallos en la gestión del Gobierno relativas a la compra de material.

Hay que profundizar, a continuación, en los resultados obtenidos sobre el protagonista en la variable actor informativo del titular para conocer más en detalle quiénes han acaparado mayor interés en los titulares.

\begin{tabular}{|c|c|c|}
\cline { 2 - 3 } \multicolumn{1}{c|}{} & Frecuencia & Porcentaje \\
\hline Culturales & 2 & $1,0 \%$ \\
\hline Económicos & 18 & $9,0 \%$ \\
\hline Institucionales/Oficiales & 41 & $20,5 \%$ \\
\hline Medios de comunicación & 3 & $1,5 \%$ \\
\hline Otros & 24 & $12,0 \%$ \\
\hline Políticos & 19 & $9,5 \%$ \\
\hline Religiosos & 1 & $0,5 \%$ \\
\hline Sanitarios & 36 & $18,0 \%$ \\
\hline Sociedad Civil & 56 & $28,0 \%$ \\
\hline Total & $\mathbf{2 0 0}$ & $\mathbf{1 0 0 , 0} \%$ \\
\hline
\end{tabular}

Tabla 3. Actor informativo del titular. Elaboración propia.

Se aprecia claramente cómo los titulares empleados en los medios digitales durante el brote de la crisis sanitaria ponen el énfasis en la sociedad civil como protagonista del foco informativo (28\%), seguido por el Gobierno e instituciones oficiales (20,5\%). En tercer lugar, los sanitarios (médicos, enfermeros, psicólogos, técnicos de emergencias) con un 18\%. Se aprecia que, con un $12 \%$, el actor otros cobra interés en los titulares. En la Tabla 4 se observa qué otros actores han cobrado relevancia para los medios en esta crisis sanitaria. 


\begin{tabular}{|c|c|c|}
\cline { 2 - 3 } \multicolumn{1}{c|}{} & Frecuencia & Porcentaje \\
\hline $\begin{array}{c}\text { Centro de Investigación } \\
\begin{array}{c}\text { Crematorios/empresas } \\
\text { funerarias }\end{array}\end{array}$ & 1 & $0,5 \%$ \\
\hline $\begin{array}{c}\text { Cifras oficiales Covid/ } \\
\text { efecto del Covid }\end{array}$ & 2 & $1,0 \%$ \\
\hline $\begin{array}{c}\text { Cuerpos y Fuerzas de } \\
\text { Seguridad del Estado }\end{array}$ & 9 & $1,0 \%$ \\
\hline Deportistas & 3 & $1,5 \%$ \\
\hline Empresarios & 4 & $2,0 \%$ \\
\hline Planeta/ Medio ambiente & 2 & $1,0 \%$ \\
\hline Periodista & 1 & $0,5 \%$ \\
\hline Subtotal & 24 & $12,0 \%$ \\
\hline $\begin{array}{c}\text { Resto de actores } \\
\text { informativos }\end{array}$ & 176 & $88,0 \%$ \\
\hline Total & $\mathbf{2 0 0}$ & $\mathbf{1 0 0 \%}$ \\
\hline
\end{tabular}

Tabla 4. Otros actores informativos. Elaboración propia.

Se puede observar cómo los Cuerpos y Fuerzas de Seguridad del Estado (45,8\%) han sido objeto de interés en los titulares que mayor alcance han obtenido. En segundo lugar, los empresarios (16,6\%), por dos aspectos fundamentales: las donaciones y las medidas económicas adoptadas durante el estado de alarma. En tercer lugar, los deportistas (12,5\%), que han mostrado su lado más solidario y comprometido para recaudar fondos para paliar los efectos económicos y sociales de esta pandemia.

En la tabla 5 se recoge el espacio informativo de los titulares. Las noticias que han conseguido mayor alcance/ engagement se han centrado fundamentalmente en el ámbito nacional (80,5\%), pero también haciéndose eco de los datos a nivel global/mundial (19\%). Por otro lado, el 3,5\% de los titulares hacían referencia a la Unión Europea y, con el mismo porcentaje (3,5\%), a noticias cuyo objeto era Estados Unidos. Asia, que es donde se ha identificado el origen de la pandemia, no ha acaparado el interés de las noticias con mayor alcance. Tan

\begin{tabular}{|c|c|c|}
\hline Asia & Frecuencia & Porcentaje \\
\hline $\begin{array}{c}\text { España (nacional, } \\
\text { autonómica, local) }\end{array}$ & 161 & $\mathbf{2 , 5} \%$ \\
\hline Estados Unidos & 7 & $3,5 \%$ \\
\hline $\begin{array}{c}\text { Oriente Medio y Magreb } \\
\text { Referencia global/ } \\
\text { mundial }\end{array}$ & 1 & $0,5 \%$ \\
\hline Unión Europea & 7 & $9,5 \%$ \\
\hline Total & $\mathbf{2 0 0}$ & $\mathbf{3 , 5 \%}$ \\
\hline
\end{tabular}

Tabla 5. Espacio informativo del titular. Elaboración propia. solo un 2,5\% de los titulares han puesto el punto atención en China.

A continuación, en la tabla 6, se ofrece un análisis del tono empleado en relación con el medio digital en el que se ha publicado la noticia.

A la vista de los resultados, se puede afirmar que larazon.es y cope.es son los medios digitales que han publicado más titulares con tono negativo (70\%), datos directamente relacionados con la línea editorial de estos medios, contraria al Gobierno al mando de la crisis. Por otro lado, lavozdegalicia.es, ha optado por titulares con un tono principalmente positivo. Se trata de un diario autonómico gallego, donde la pandemia golpeó de manera leve y afín al Gobierno regional, lo que explica estos datos. La mayoría de los medios, a excepción de cope.es y sevilla.abc.es, han balanceado simétricamente entre el tono negativo y positivo. En el caso de cope.es, la asimetría viene determinada por predominar titulares negativos, mientras que el medio sevilla.abc.es ha destacado por el tono positivo en sus titulares, con una diferencia abultada con respecto a los negativos, lo que puede deberse también a la evolución favorable de la pandemia en esta Comunidad.

En la tabla 7 se analiza la finalidad/tono del titular con respecto a las temáticas. Sanidad ha sido objeto del mayor número de titulares en negativo y positivo; por lo tanto, existe un equilibrio en el objeto principal temático en el que se han centrado las noticias objeto de estudio con respecto a la crisis del coronavirus. Así mismo, se aprecia que la política ha sido el segundo tema que más titulares con tono negativo ha obtenido. Por otra parte, los temas que se han abordado de manera positiva en los titulares, por este orden de importancia, han sido sanidad, economía y política. Estos tres ejes temáticos coinciden con los sectores más afectados por la crisis, por lo que eran focos de interés para los lectores. Teniendo en cuenta que los medios los proponen desde una óptica positiva o negativa, en lugar de neutral, lo que consiguen es potenciar su alcance/engagement, imprimiéndole una emoción que empuja al usuario a leerlo y compartirlo.

Por último, en la tabla 8 se analiza el tono empleado en los titulares con respecto a los actores informativos de esta crisis sanitaria.

El análisis pormenorizado de los titulares negativos relacionados con sociedad civil hace referencia a los fallecidos, temas de violencia de género, no respetar las medidas de confinamiento y pérdida de familiares queridos. En lo que respecta a institucionales/oficiales, hacen referencia a una gestión mejorable del Gobierno con respecto a la crisis, conocimiento por parte del 


\begin{tabular}{|c|c|c|c|c|c|c|}
\hline & & & \multicolumn{3}{|c|}{ Finalidad/Tono del titular } & \multirow{2}{*}{ Total } \\
\hline & & & Negativo & Neutro & Positivo & \\
\hline \multirow{26}{*}{ Medio digital } & \multirow{2}{*}{ 20minutos.es } & Recuento & 8 & 6 & 6 & 20 \\
\hline & & \% dentro de Medio digital & $42,1 \%$ & $31,6 \%$ & $26,3 \%$ & $100,0 \%$ \\
\hline & \multirow{2}{*}{ abc.es } & Recuento & 8 & 1 & 11 & 20 \\
\hline & & $\%$ dentro de Medio digital & $40,0 \%$ & $5,0 \%$ & $55,0 \%$ & $100,0 \%$ \\
\hline & \multirow{2}{*}{ cope.es } & Recuento & 7 & 1 & 2 & 10 \\
\hline & & \% dentro de Medio digital & $70,0 \%$ & $10,0 \%$ & $20,0 \%$ & $100,0 \%$ \\
\hline & \multirow{2}{*}{ eldiario.es } & Recuento & 12 & 3 & 5 & 20 \\
\hline & & \% dentro de Medio digital & $60,0 \%$ & $15,0 \%$ & $25,0 \%$ & $100,0 \%$ \\
\hline & \multirow{2}{*}{ elmira.es } & Recuento & 1 & 6 & 3 & 10 \\
\hline & & $\%$ dentro de Medio digital & $10,0 \%$ & $60,0 \%$ & $30,0 \%$ & $100,0 \%$ \\
\hline & \multirow{2}{*}{ elmundo.es } & Recuento & 6 & 4 & 10 & 20 \\
\hline & & $\%$ dentro de Medio digital & $30,0 \%$ & $20,0 \%$ & $50,0 \%$ & $100,0 \%$ \\
\hline & \multirow{2}{*}{ europapress.es } & Recuento & 4 & 7 & 9 & 20 \\
\hline & & $\%$ dentro de Medio digital & $20,0 \%$ & $35,0 \%$ & $45,0 \%$ & $100,0 \%$ \\
\hline & \multirow{2}{*}{ farodevigo.es } & Recuento & 1 & 7 & 2 & 10 \\
\hline & & \% dentro de Medio digital & $10,0 \%$ & $70,0 \%$ & $20,0 \%$ & $100,0 \%$ \\
\hline & \multirow{2}{*}{ lavozdelalicia.es } & Recuento & 3 & 1 & 6 & 10 \\
\hline & & $\%$ dentro de Medio digital & $30,0 \%$ & $10,0 \%$ & $60,0 \%$ & $100,0 \%$ \\
\hline & \multirow{2}{*}{ lazaron.es } & Recuento & 7 & 1 & 2 & 10 \\
\hline & & \% dentro de Medio digital & $70,0 \%$ & $10,0 \%$ & $20,0 \%$ & $100,0 \%$ \\
\hline & \multirow{2}{*}{ publico.es } & Recuento & 3 & 6 & 1 & 10 \\
\hline & & \% dentro de Medio digital & $30,0 \%$ & $60,0 \%$ & $10,0 \%$ & $100,0 \%$ \\
\hline & \multirow{2}{*}{ sevilla.abc.es } & Recuento & 5 & 5 & 10 & 20 \\
\hline & & \% dentro de Medio digital & $25,0 \%$ & $25,0 \%$ & $50,0 \%$ & $100,0 \%$ \\
\hline & \multirow{2}{*}{ telecinco.es } & Recuento & 6 & 10 & 5 & 21 \\
\hline & & \% dentro de Medio digital & $28,6 \%$ & $47,6 \%$ & $23,8 \%$ & $100,0 \%$ \\
\hline \multirow{2}{*}{\multicolumn{2}{|c|}{ Total }} & Recuento & 71 & 58 & 71 & 200 \\
\hline & & $\%$ dentro de Medio digital & $35,5 \%$ & $29,0 \%$ & $35,5 \%$ & $100,0 \%$ \\
\hline
\end{tabular}

Tabla 6. Tabla cruzada de variables tono con medio digital. Elaboración propia.

Gobierno de los efectos devastadores desde enero y la denuncia que ha recibido el Presidente del Gobierno ante el Tribunal Supremo. En lo referido a sanitarios, los titulares negativos hacen referencia al contagio y muerte de estos profesionales, a la falta de solidaridad de los vecinos que no quieren que habiten durante la pandemia en sus casas y la falta de equipos de protección para ejercer su profesión de forma segura.

En cuanto al tono positivo de la sociedad civil, hace énfasis en medidas de solidaridad (donaciones económicas y de material sanitario) y compromiso de la sociedad civil con los más vulnerables. También se reflejan otros ítems como las primeras altas de pacientes que han vencido al SARS-CoV-2, así como las primeras noticias de descenso de contagios y muertes entre la población. Con respecto a los aspectos positivos más destacados en la categoría de institucionales/oficiales, los medios se hacen eco de las políticas relacionadas con las ayudas para paliar el impacto del coronavirus y posibles medidas de relajamiento de los acuerdos adoptados en el estado de alarma para la desescalada. En cuanto a los aspectos positivos referidos al actor informativo económico, están en consonancia con medidas de impulso socioeconómico por parte de empresas e instituciones públicas, a saber: mantenimiento de suministros básicos a pesar de los impagos, donación de empresas de material sanitario para hospitales y sanciones económicas cuantiosas para quienes incumplan el estado de alarma.

En último lugar, se analizó la redundancia de conceptos presentes en los titulares a partir del recuento de palabras realizado en Atlas.ti, de modo que pudieran 


\begin{tabular}{|c|c|c|c|c|c|}
\hline & & & \multicolumn{3}{|c|}{ Finalidad/Tono del titular } \\
\hline & & & Positivo & Negativo & Neutro \\
\hline \multirow{12}{*}{$\begin{array}{l}\text { Tema del } \\
\text { titular }\end{array}$} & Bienestar social (políticas sociales) & Recuento & 2 & 0 & 2 \\
\hline & Deportes & Recuento & 0 & 1 & 0 \\
\hline & Economía & Recuento & 6 & 2 & 14 \\
\hline & Empleo & Recuento & 2 & 3 & 1 \\
\hline & Industria & Recuento & 0 & 2 & 1 \\
\hline & Internacional & Recuento & 1 & 0 & 0 \\
\hline & Medio Ambiente & Recuento & 1 & 0 & 1 \\
\hline & Monarquía & Recuento & 1 & 0 & 0 \\
\hline & Poder Judicial & Recuento & 0 & 2 & 1 \\
\hline & Política & Recuento & 16 & 8 & 9 \\
\hline & Religión & Recuento & 0 & 0 & 1 \\
\hline & Sanidad & Recuento & 42 & 40 & 41 \\
\hline \multirow{2}{*}{\multicolumn{2}{|c|}{ Total }} & Recuento & 71 & 58 & 71 \\
\hline & & $\%$ dentro de Tema del titular & $35,5 \%$ & $29,0 \%$ & $35,5 \%$ \\
\hline
\end{tabular}

Tabla 7. Tabla cruzada de variables tono con tema del titular. Elaboración propia.

identificarse los ejes discursivos implícitos en el uso de los términos en los titulares (Figura 1).

Entre los diez términos más repetidos en los titulares, destacan tres bloques: el virus y sus consecuencias, los protagonistas y los lugares de actualidad en relación con la pandemia. En el primero de los bloques, se integran COVID-19, Coronavirus, Fallecidos (agrupación de fallece/n, fallecimiento, muere/n, muerto/s, fallecidos, muertes, murió), Años (término asociado a la edad de los fallecidos y, en menor proporción, de ancianos recuperados) y Donaciones (agrupación de dona, donará, donan/en, donantes) realizadas para paliar sus efectos. En el segundo de los ejes, los protagonistas que se erigen son el Gobierno (agrupación de Gobierno, Sánchez,
Presidente) y los Sanitarios (agrupación de médico/a/s, sanitario/s, enfermero/as, doctora). En último lugar, Madrid y China (agrupación de China, Wuhan, Asia) se reflejan en los titulares como ubicaciones principales de la crisis sanitaria.

\section{Conclusiones}

A partir de los datos obtenidos, es posible extraer las algunas conclusiones derivadas del tratamiento informativo del SARS-CoV-2:

- Las noticias de la pandemia del SARS-CoV-2 con mayor alcance/engagement han gravitado fundamentalmente sobre aspectos sanitarios, políticos

\begin{tabular}{|c|c|c|c|c|c|}
\hline & & & \multicolumn{3}{|c|}{ Finalidad/Tono del titular } \\
\hline & & & Positivo & Negativo & Neutro \\
\hline \multirow{9}{*}{$\begin{array}{c}\text { Actor } \\
\text { informativo } \\
\text { del titular }\end{array}$} & Culturales & Recuento & 1 & 0 & 1 \\
\hline & Económicos & Recuento & 5 & 3 & 10 \\
\hline & Institucionales/Oficiales & Recuento & 18 & 9 & 14 \\
\hline & Medios de comunicación & Recuento & 2 & 1 & 0 \\
\hline & Otros & Recuento & 8 & 9 & 7 \\
\hline & Políticos & Recuento & 9 & 5 & 5 \\
\hline & Religiosos & Recuento & 0 & 0 & 1 \\
\hline & Sanitarios & Recuento & 10 & 17 & 9 \\
\hline & Sociedad Civil & Recuento & 18 & 14 & 24 \\
\hline \multirow{2}{*}{\multicolumn{2}{|c|}{ Total }} & Recuento & 71 & 58 & 71 \\
\hline & & $\%$ dentro de Actor informativo del titular & $35,5 \%$ & $29,0 \%$ & $35,5 \%$ \\
\hline
\end{tabular}

Tabla 8. Tabla cruzada de variables tono con actor informativo del titular. Elaboración propia. 


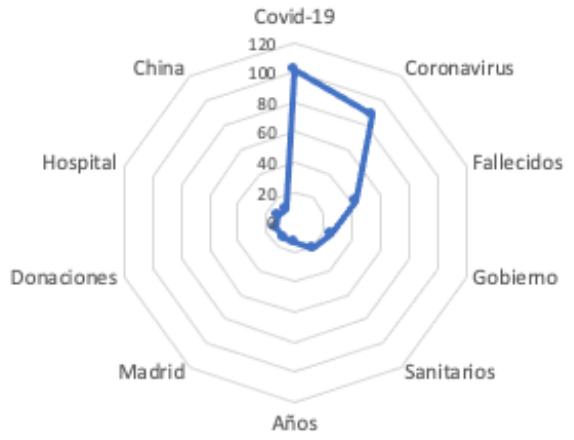

Figura 1. Conceptos redundantes en el discurso del titular. Elaboración propia

(gestión de la crisis y medidas adoptadas en el estado de alarma) y económicos (ayudas para paliar los efectos devastadores de la crisis sanitaria). Estos datos entroncan directamente con la UyG, que postula que los usuarios buscan noticias que le permitan satisfacer su necesidad de orientación psicológica (Blumler, 1979; Katz, Haas y Gurevitch, 1973). Esto se ha visto potenciado en el caso que nos ocupa: una crisis sanitaria sin precedentes, protagonizada por un virus desconocido. La pandemia generó mucha ansiedad en la población, impulsando su necesidad de recurrir a las noticias para resolver dudas y mantener el contacto con el exterior en pleno confinamiento.

- En cuanto al tono empleado, se observa una simetría absoluta en las noticias que han obtenido más alcance/engagement: presentan mayoritariamente titulares positivos o negativos. Esta polarización tiene su explicación en la relación entre la carga emocional y la viralidad: este tipo de mensajes son más propensos a recibir clics de los lectores (Berger y Milkman, 2012).

- La sociedad civil ha sido el actor informativo que ha suscitado mayor interés de los medios digitales como protagonista destacado de esta crisis sanitaria, por delante del Gobierno e, incluso, de los propios sanitarios. Cabe destacar el papel relevante que han reservado los titulares de las noticias a las Fuerzas y Cuerpos de Seguridad del Estado, los empresarios y los deportistas (todos ellos mostrando la faceta más solidaría y comprometida con la sociedad). De alguna manera, todo ello está en consonancia con la conclusión primera, donde los titulares gravitaban en torno a la sanidad, la política y la economía, por lo que los actores tienen relación directa con esos grandes bloques temáticos.

- Las noticias que mayor alcance/engagement han obtenido han centrado el interés de la crisis fundamentalmente en lo concerniente al ámbito nacional (autonómico, regional y local), por lo su éxito vendría explicado por la UyG: estos contenidos satisfacen la necesidad de los usuarios de familiarizarse con el entorno a través de los medios de comunicación (Matthes, 2005; Weaver, 1980).

\section{Referencias}

Amoedo, A., Vara-Miguel, A., y Negredo, S. (2018). Digital news report. es 2018. Una audiencia diversa y preocupada por la desinformación. Center for Internet Studies and Digital Life. Universidad de Navarra.

Bardin, L. (1986). El análisis de contenido. Akal.

Berelson, B. (1952). Content analysis in communication research. Hafner.

Berger, J., y Milkman, K. (2012). What makes online content viral? Journal of marketing research, 49(2), 192-205. https://doi. org/10.1509/jmr.10.0353

Blumler, J. G. (1979). The role of theory in uses and gratifications studies. Communication Research, 6, 9-36. https://doi. org/10.1177/009365027900600102

Boczkowski, P. J., Mitchelstein, E., y Matassi, M. (2018). 'News comes across when I'm in a moment of leisure': Understanding the practices of incidental news consumption on social media. New media 8 society, 20(10), 3523-3539. https://doi.org/10.1177/1461444817750396

Bowden, J. (2009). The Process of Customer Engagement: A Conceptual Framework. The Journal of Marketing Theory and Practice, 17(1), 63-74. https://doi.org/10.2753/MTP1069-6679170105

Campos-Freire, F. (2010). Los nuevos modelos de gestión de las empresas mediáticas. Estudios sobre el mensaje periodístico, 16, 13-30.

Casero-Ripollés, A. (2020). Impacto del Covid-19 en el sistema de medios. Consecuencias comunicativas y democráticas del consumo de noticias durante el brote. El Profesional de la Información, 29(2). https://doi.org/10.3145/epi.2020.mar.23

Castells, M. (2011). Comunicación y Poder. Alianza.

Culver, S., y Jacobson, T. (2012). Alfabetización mediática como método para fomentar la participación cívica. Comunicar: Revista científica iberoamericana de comunicación y educación, 20(39), 73-80. https://doi.org/10.3916/C39-2012-02-07

Deltell, L. (2014). Audiencia social versus audiencia creativa: caso de estudio Twitter. Estudios sobre el Mensaje Periodístico, 20(1), 33-47. https://doi.org/10.5209/rev_ESMP.2014.v20.n1.45217

Dessart, L. (2017). Social media engagement: a model of antecedents and relational outcomes. Journal of Marketing Management, 33(5-6), 375-399. https://doi.org/10.1080/0267257X.2017.1302975

Dutta-Bergman, M. J. (2004). Complementarity in consumption of news types across traditional and new media. Journal of broadcasting \& electronic media, 48(1), 41-60. https://doi.org/10.1207/ s15506878jobem4801_3

El consumo de noticias, la categoría de internet que más sube durante el confinamiento por coronavirus (29 de abril 2020). ABC. https://www.abc.es/tecnologia/redes/abci-consumo-noticias-categoria-internet-mas-sube-durante-confinamiento-coronavirus-202004280154_noticia.html

Effing, R., Van Hillegersberg, J., y Huibers, T. (2011). Social media and 
political participation: are Facebook, Twitter and YouTube democratizing our political systems? En E. Tambouris, A. Macintosh y $\mathrm{H}$. de Bruijn (eds), Electronic Participation. ePart 2011. Lecture Notes in Computer Science (pp. 25-35). Springer.

Fernández-Medina, F. J., Proust, V., y Núñez-Mussa, E. (2018). Consumo incidental de noticias en un contexto de redes sociales y múltiples pantallas. Revista Ibérica de Sistemas e Tecnologias de Informação, E16, 308-320. http://www.risti.xyz/issues/ristie16.pdf

Gambetti, R. C., Graffigna, G., y Biraghi, S. (2012). The grounded theory approach to consumer-brand engagement: The practitioner's standpoint. International Journal of Market Research, 54(5), 659-687. https://doi.org/10.2501/IJMR-54-5-659-687

Guallar, J. (2011). Prensa digital en 2010. Anuario ThinkEPI, 5, 101-105. https://bit.ly/3gi4nlN

Hernández-Pérez, T., y Rodríguez Mateos, D. (2016). Medición integral de las audiencias: sobre los cambios en el consumo de información y la necesidad de nuevas métricas en medios digitales. Hipertext.net, 14. https://doi.org/10.2436/20.8050.01.32

Hípola, G. (2017). La audiencia creativa: La interacción a través del infoentretenimiento. Más Poder Local, 32, 24-25. https://bit.ly/2Uj6KML

Jenkins, H. (2006). Fans, bloggers, and gamers: Exploring participatory culture. NYU Press.

Katz, E., Blumler, J. G., y Gurevitch, M. (1973). Uses and gratifications research. The public opinion quarterly, 37(4), 509-523. https://doi. org/10.1086/268109

Katz, E., Haas, H., y Gurevitch, M. (1973). On the use of the mass media for important things. American Sociological Review, 38(2), 164-181. https://doi.org/10.2307/2094393

Krippendorff, K. (1990). Metodología del análisis de contenido. Teoría y Práctica. Paidós.

Labella, L. I. (2012). Saturación informativa: la multiplicación de la oferta a través de Internet no aumenta el consumo de noticias. Razón y Palabra, 81, 59-18. http://www.razonypalabra.org.mx/N/N81/ V81/38_Izquierdo_V81.pdf

Lee Cook, J. (2009). Incidental Exposure to News: Limiting Fragmentation in the New Media Environment [Tesis doctoral, University of Texas]. http://hdl.handle.net/2152/6686

Liang, C. C., y Wu, R. Y. (s.f.). A pilot study of the impact of emotions to exaggerated news title. https://bit.ly/2MxHxcY

López-Aranguren, E. (2016). El análisis de contenido tradicional. En M. García Ferrando, J. Ibáñez y F. Alvira (coords.), El análisis de la realidad social: Métodos y técnicas de investigación (pp. 594-616). Alianza.

Martín López, E. (1963). El análisis de contenido. Revista de Estudios Políticos, 132, 45-64. https://bit.ly/3cCK2oY

Martínez-Costa, M. D. P., Sánchez-Blanco, C., y Serrano-Puche, J. (2018). El consumo de noticias online en España: temáticas, formatos e itinerarios de acceso. En J. L. González-Esteban y J. A. García-Avilés (coords.), Mediamorfosis: radiografía de la innovación en el periodismo (pp. 35-60). Universidad Miguel Hernández.

Matthes, J. (2005). The need for orientation towards news media: Revising and validating a classic concept. International Journal of Public Opinion Research, 18(4), 422-444. https://doi.org/10.1093/ ijpor/edh118

Medios digitales y televisión, principales opciones de entrete- nimiento (24 de marzo 2020). Economiadigital.es. https://bit. ly/2Z9PkTW

La prensa digital dispara su consumo por el Covid-19 (17 de marzo 2020). MarketingNews. https://bit.ly/3bXPMKg

Mersey, R. D., Malthouse, E. C., y Calder, B. J. (2010). Engagement with online media. Journal of Media Business Studies, 7(2), 39-56. https:// doi.org/10.1080/16522354.2010.11073506

Mitchelstein, E., y Boczkowski, P. J. (2018). Juventud, estatus y conexiones. Explicación del consumo incidental de noticias en redes sociales. Revista mexicana de opinión pública, 24, 131-145. https://doi. org/10.22201/fcpys.24484911e.2018.24.61647

Newman, N., Fletcher, R., Kalogeropoulos, A., y Nielsen, R. K. (2019). Reuters institute digital news report 2019 (Vol. 2019). Reuters Institute for the Study of Journalism. https://bit.ly/3dF8J5x

Ng, Y. L., y Zhao, X. (2020). The human alarm system for sensational news, online news headlines, and associated generic digital footprints: A uses and gratifications approach. Communication Research, 47(2), 251-275. https://doi.org/10.1177/0093650218793739

Oeldorf-Hirsch, A., y Sundar, S. S. (2015). Posting, commenting, and tagging: Effects of sharing news stories on Facebook. Computers in Human Behavior, 44, 240-249. https://doi.org/10.1016/J. CHB.2014.11.024

Piñuel, J. L., y Gaitán, J. A. (1995). Metodología general: Conocimiento científico e investigación en la comunicación social. Síntesis.

Rubin, A. M. (2009). The uses-and-gratifications perspective on media effects. En J. Bryant y M. B. Oliver (eds.), Media effects: Advances in theory and research (pp. 525-548). Routledge.

Serrano, M. J. H., Arellano, P. R., Graham, G., y Greenhill, A. (2017). Del prosumidor al prodiseñador: el consumo participativo de noticias. Comunicar: Revista científica iberoamericana de comunicación y educación, 25(50), 77-88. https://doi.org/10.3916/C50-2017-07

Sundar, S. S., y Limperos, A. M. (2013). Uses and grats 2.0: New gratifications for new media. Journal of Broadcasting and Electronic Media, 57(4), 504-525. https://doi.org/10.1080/08838151.2013.845827

Televisión y medios digitales, principales opciones elegidas durante el confinamiento según un estudio (23 de marzo 2020). Europapress. https://bit.ly/2KUmi45

Weaver, D. H. (1980). Audience need for orientation and media effects. Communication Research, 7(3), 361-373. https://doi.

org/10.1177/009365028000700305

CV

Natalia Abuín-Vences. Es Doctora en Comunicación Audiovisual y Publicidad por la Universidad Complutense de Madrid. Actualmente es profesora de Cultura de las Organizaciones en el Departamento de Ciencias de la Comunicación Aplicada de la UCM. En 2015 fue miembro del Comité de Innovación de Ofertas Formativas Digitales ANECA-SETSI. Ha participado en diversos programas y proyectos de investigación de índole nacional (Plan Nacional $\mathrm{I}+\mathrm{D}+\mathrm{i}$ ), autonómico (Comunidad de Madrid) e 
internacional (a través de la European Communication Research and Education Association).

Javier Sierra-Sánchez. Es Doctor en Ciencias de la Información por la Universidad Complutense de Madrid (UCM) y licenciado en Comunicación Audiovisual y Periodismo por la misma universidad. Master oficial en Marketing y Comunicación Corporativa por la Universidad San Jorge. Experto en RRPP Internacionales por la UCM. Experto en Protocolo y Ceremonial del Estado e Internacional por la Universidad de Oviedo y la Escuela Diplomática de Madrid. Actualmente ejerce docencia como profesor contratado doctor en la Universidad Complutense de Madrid. Su índide H en Google Scholar es superior a 10

Luis Mañas-Viniegra. Es profesor e investigador en el Departamento de Ciencias de la Comunicación Aplicada de la Universidad Complutense de Madrid. Es Doctor en Comunicación Audiovisual y Publicidad con Premio Extraordinario por la misma Universidad. Ha sido profesor en las universidades Carlos III de Madrid, Rey Juan Carlos y Universidad de Valladolid. Es miembro del Grupo de Investigación Complutense de Gestión de Marca y Comunicación Integrada.

Patricia-Núñez Gómez. Es Doctora en Comunicación Audiovisual y Publicidad, Profesora Titular y Directora del Departamento de Ciencias de la Comunicación Aplicada de la Universidad Complutense de Madrid. También es Directora de la Cátedra Extraordinaria de Marketing y Comunicación para la Infancia y la Adolescencia de la misma Universidad.

осм Observatorio de Cibermedios

\section{https://observatoriocibermedios.upf.edu/}

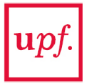
Universitat Pompeu Fab
Barcelona

Departamento de Comunicación
Grupo DigiDoc

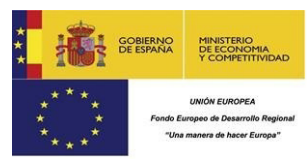

El Observatorio de Cibermedios es una producción del Grupo de Investigación en Documentación Digital y Comunicación Interactiva (DigiDoc) del Departamento de Comunicación de la Universitat Pompeu Fabra.

El Observatorio de Cibermedios (OCM) forma parte del proyecto del Plan Nacional "Narración interactiva y visibilidad digital en el documental interactivo y el periodismo estructurado". RTI2018-095714-B-C21 (MINECO/FEDER), Ministerio de Ciencia, Innovación y Universidades (España). 